\title{
QUANTIFICAÇÃO DA GEODIVERSIDADE E AVALIAÇÃO DA DISTRIBUIÇÃO ESPACIAL NA BACIA HIDROGRÁFICA DO RIBEIRÃO DO SILVA
}

\author{
Patrícia Pascoal Goulart*
}

\begin{abstract}
Resumo
O termo Geodiversidade tem sido empregado na referência ao meio físico, abrangendo os elementos abióticos como rochas, formas de relevo, solos e rio. Esses elementos, quando combinados com a biodiversidade, configuram ambientes físicos que permitem uma análise quantitativa espacial. A pesquisa de geodiversidade e avaliação da distribuição espacial na bacia do Silva foi desenvolvida através do modo indireto, utilizando uma vigorosa base de dados geográficos e programas computacionais de análise espacial e estatística, que permitiram alcançar um índice de riqueza dos elementos abióticos. As diferentes categorias dos índices de geodiversidade foram agrupadas, respeitando a homogeneidade entre as classes, através do algoritmo "K-means". Os resultados obtidos foram grupos homogêneos com similaridade entre a quantidade de ocorrência das variáveis, que podem fundamentar as tomadas de decisão para a geoconservação.
\end{abstract}

Palavras-chave:métodos estatísticos, índice de geodiversidade, distribuição espacial.

\begin{abstract}
The Geodiversityhasbeenused in referencetotheenvironment, includingtheabioticelements rocks, landforms, soilsandriver. Theelementsofgeodiversity, whencombinedwithbiodiversity, configure physicalenvironmentsthatallow a quantitativeanalysisspace. The researchofgeodiversityandspatialdistribution in thewatershedof Silva wasdevelopedthroughanindirectway, using a strong base geographic data andcomputerprograms for spatialanalysis, whichhadachievedan index ofwealthofabioticelements. The different index categoriesweregroupedgeodiversity, respectingthehomogeneitybetweenclasses, throughof cluster analysis - K-means. The resultwastheclassificationofgroupswithdifferentlevelsofthegeodiversity.
\end{abstract}

Keywords:statisticalmethods, index ofgeodiversity, spatialdistribution

\footnotetext{
*1Geógrafa, Discente do mestrado em Análise e modelagem de sistemas ambientais. goulart.ppatricia@gmail.com 


\section{1-INTRODUÇÃO}

O termo Geodiversidade tem sido empregado na referência ao complexo físico dapaisagem, abrangendo os elementos abióticos como rochas, formas de relevo, solos e rios, (MANOSSO, 2012). Os diferentes elementos da geodiversidade e da biodiversidade, quando combinados, configuram ambientes físicos que permitem uma análise quantitativa espacial.

Segundo GRAY (GRAY, 2004 in MANOSSO, 2012), a geodiversidade deve ser entendida a partir de um conjunto de valores, divididos entre valor intrínseco, cultural, estético, econômico, funcional, científico e educativo.

Portanto, diante das variadas formas de ocupação e exploração dos recursosnaturais, tanto o conteúdo da geodiversidade, bem como os seus valores, encontram-se sob risco ou ameaçados. Nesse sentido, é interessante a sensibilidade através de elementos e processos, que através da atividade humana podem alterar a geodiversidade.

De acordo com BRILHA (BRILHA, 2005), do mesmo modo que abiodiversidade, como se perdem as espécies bióticas ainda não estudadas completamente, elementos da geodiversidade também podem se perder, muitas vezes pelo desconhecimento sobre a distribuição espacial, conteúdo e importância.

A geoconservação pode ser compreendida como a preservação da diversidadenatural, de elementos e processos geológicos, geomorfológicos, sistemas de solos e manutenção de valores e processos. Para a efetiva geoconservaçãoé necessária a implantação do gerenciamento de conteúdo e valores da geodiversidade e de seu patrimônio, através do estabelecimento de políticas eficazes de proteção.

Em conformidade com os conceitos apresentados, o objetivo deste estudo é a quantificação da geodiversidade da bacia do ribeirão do Silva. A área está inserida no quadrilátero ferrífero, possui riqueza de recursos minerais e paisagísticos, podendo ser identificado o potencial para a exploração mineral, atividade que é altamente conflituosa quando relacionada à conservação do patrimônio geológico.

A análise por aglomeração, através do algoritmo K-means, foi necessária para agrupar conjuntos de categorias em grupos homogêneos, observando apenas as similaridades ou dissimilaridades entre elas, em conformidade como o conceito de Hair et al (2006)“Análise de 
cluster é um grupo de técnicas multivariadas cujo principal objetivo é agrupar objetos a partir de suas características" (HAIR et al, 2006, in FILHO et al, 2012).

\section{Estudo de Caso: A Bacia Hidrográfica do Ribeirão do Silva}

A bacia hidrográfica do ribeirão do Silva está situada na porção oeste do município de Itabirito, localizado a 60 quilômetros a sudeste de Belo Horizonte, ocupandouma área de 95,080 $\mathrm{Km}^{2}$, com aproximadamente $20 \mathrm{Km}$ de extensão (Figura 1). O ribeirão doSilva, um dos principais afluentes do rio Itabirito, está situado no interior do sinclinal Moeda,ao sul do platô Moeda, transformando-se depois em ribeirão Mata Porcos e drenando para oeixo sinclinal em direção sul, estrangulado entre as serras da Moeda e das Serrinhas, (IBGE1985). Seu acesso se dá pela BR-040, partindo de Belo Horizonte em direção ao Rio de Janeiro.

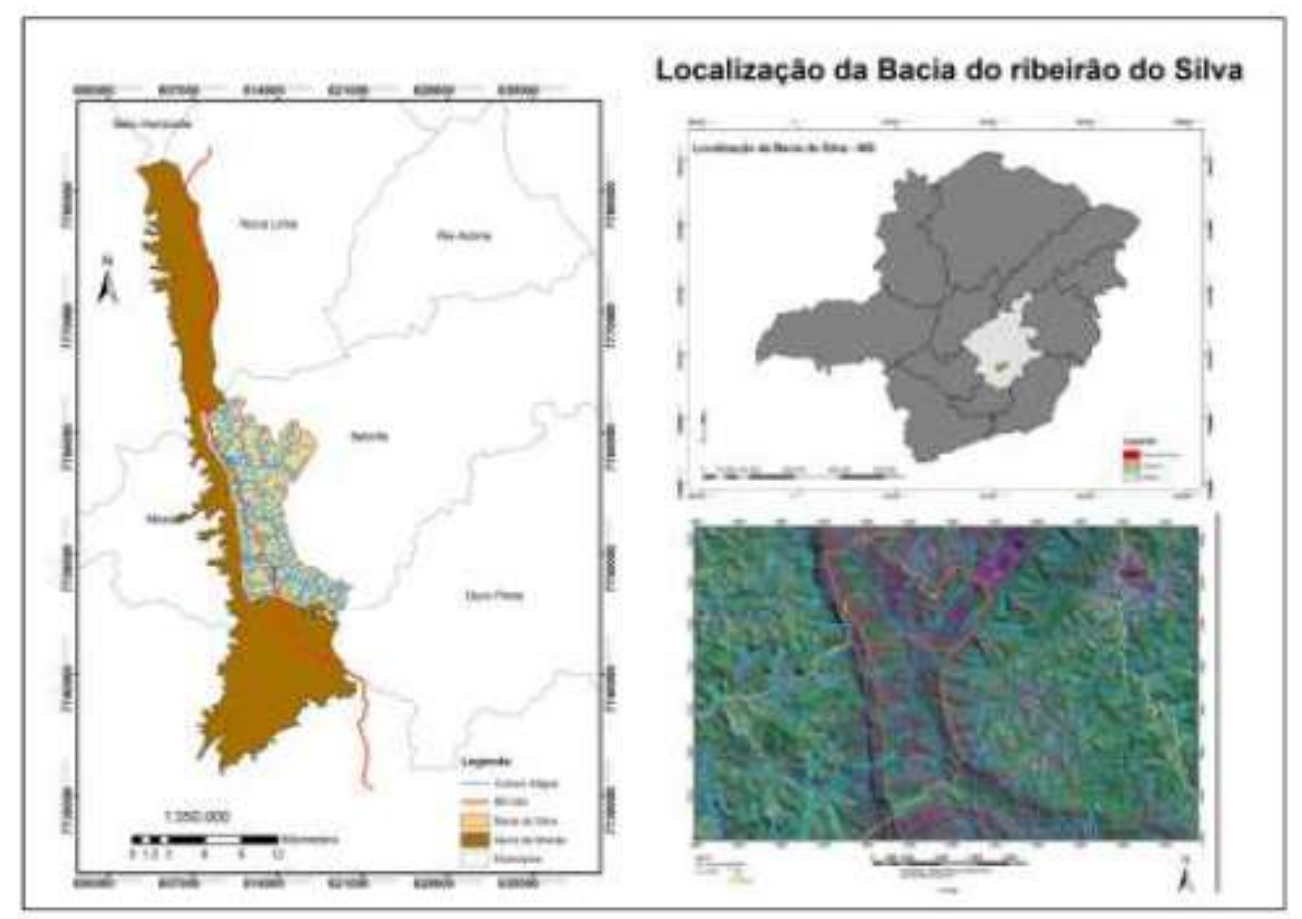

Figura 1: Localização do Ribeirão do Silva

No que tange aos aspectos físicos, a bacia do ribeirão do Silva, está inserida na região do Quadrilátero Ferrífero, o que lhe confere uma marcante diversidade litológica representada principalmente por itabiritos, quartzitos, dolomitos, filitos, xistos, granitos e metaarenitos, (SOLÁ, 2008). Essas formações que compõem a estrutura do Sinclinal Moeda possuem alta 
capacidade aquífera, capazes de acumular consideráveis quantidades de água e manter as vazões de base dos cursos d'água.

O relevo na bacia hidrográfica do Ribeirão do Silva é caracterizado por apresentar uma unidade de paisagem ondulada na porção intermontana e montanhoso com vertentes dedeclividade acentuada nas porções da serra da Moeda e Serrinhas. A altitude varia entre 980 e 1560 metros, apresentando uma amplitude de aproximadamente 580 metros.

O meio natural regional apresenta rica biodiversidade com potencial para existência defauna e flora endêmica. Os aspectos morfológicos associados à diversificada litologia, permitiu o desenvolvimento de exuberante biodiversidade, associada a uma paisagem singular. Há diversos tipos de ecossistemas na área, destacando a Floresta Estacional Semidecidual - um dos tipos da Mata Atlântica - acompanhando as linhas de drenagem; Cerrado na média encosta; e Campo Rupestre nas cristas e sobre afloramentos rochosos.

As áreas protegidas na bacia do Silva são a Estação Ecológica de Aredes e o Monumento Natural da Serra da Moeda. Sob a ótica patrimonial, o Monumento Natural daSerra da Moeda apresenta como um grande atrativo geológico, histórico e cultural.

As atividades econômicas na área de estudo são representadas pelas atividades de exploração mineral, de ferro, argila e beneficiamento de água mineral. O comércio e as atividades agrosilvopastoris se apresentam de modo incipiente. Cabe ressaltar que $70 \%$ daárea pertencem às mineradoras. Além da população nativa, a bacia do ribeirão do Silva, éocupada por moradores dos condomínios Aconchego da Serra e Vila Bela e também por invasores. A população da bacia é atendida pelas concessionárias de água, luz e telefone. Comrelação ao sistema viário, o principal acesso à bacia éa rodovia BR-040 que liga Minas Gerais ao Rio de Janeiro.

Segundo o Plano Diretor de Itabirito, elaborado em 2005, a área onde se insere a bacia do ribeirão do Silva, compreende as zonas de mineração, rural e de preservação. Os principais projetos em desenvolvimento referem-se à expansão mineraria; residencial; industrial (reservas de água mineral) e à criação de um segundo Distrito Industrial do município de Itabirito. Segundo o Zoneamento Ecológico e Econômico de Minas Gerais - ZEE-MG, (MINAS GERAIS, 2009) a área onde se insere a bacia hidrográfica do ribeirão do Silva éindicada como área prioritária para conservação, em função de sua alta vulnerabilidade natural.

Em conformidade com a caracterização da área, foi verificada a pertinência de realizar uma análise quantitativa da geodiversidade local. A bacia hidrográfica apresenta grande 
diversidade de recursos, porém, até a atualidade os estudos realizados apresentam, em grandemaioria, a análise qualitativa da biodiversidade ou do meio físico.

\section{Metodologia empregada}

Para a interpretação da distribuição espacial da geodiversidade por meio do índice de riqueza e a abundância relativa é necessário utilizar um recorte espacial para aplicação em células amostrais pré definidas (MANOSSO 2012). A avaliação quantitativa e de distribuiçãoespacial é fundamentada na frequência e diversidade do conjunto de variáveis, feições geológicas ou da paisagem, que permitem quantificar e comparar diferentes áreas.

A pesquisa de geodiversidade e avaliação da distribuição espacial na bacia do Silva,tem como embasamento a fundamentação teórica proposta por XAVIER- DA- SILVA (XAVIER DA SILVA, 2004), que permite alcançar um índice de riqueza dos elementos domeio físico, através de método indireto de quantificação, ou seja, sem levantamento de campo. Ainda, conforme o autor, a geodiversidade pode ser mensurada em cinco índices: Geodiversidade Específica: corresponde ao número de classes que cada variável éencontrada em um dado polígono.

Geodiversidade de Posição: é a ordenação dos polígonos de acordo com a geodiversidade de cada um dos grupos, do menos diverso ao mais diverso.

Geodiversidade Múltipla: consiste na soma simples de todas as classes de todas as variáveis em um mesmo polígono.

Geodiversidade Múltipla Ponderada: é a divisão do índice de geodiversidade múltipla pela área do polígono, isso permite uma comparação entre polígonos de diferentes tamanhos.

Geodiversidade de Posição Múltipla: ordenação dos polígonos de acordo com a geodiversidade múltipla ponderada de cada um dos grupos, do menos diverso ao mais diverso.

SERRANO E RUIZ FLAÑO (SERRANO, 2007 in MANOSSO, 2012), separam os elementos que compõem a geodiversidade associados à topografia, geologia, geomorfologia,hidrologia e solos. A figura 2 ilustra o processo de escolha do agrupamento de dados para aquantificação e classificação dos índices. 


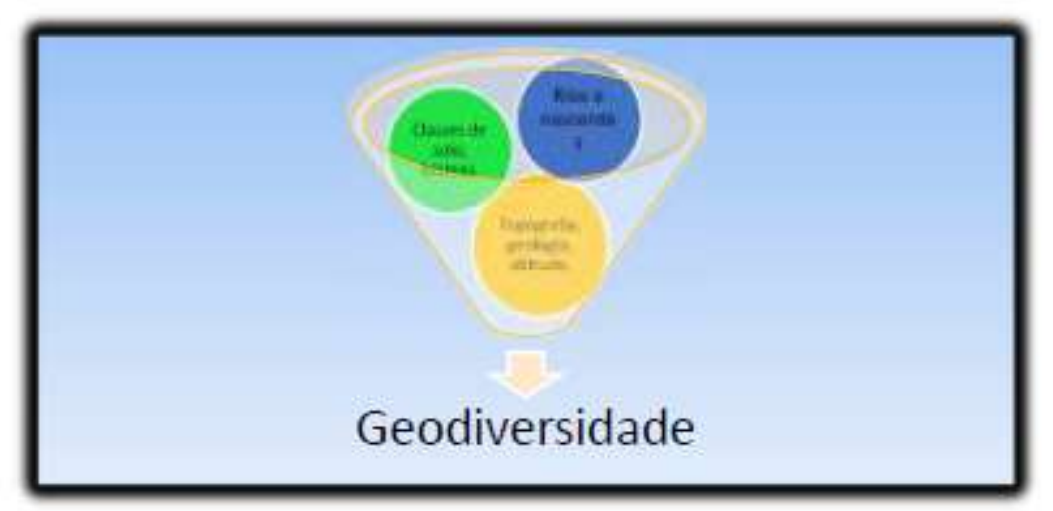

Figura 2: construção dos índices de geodiversidade

Em conformidade com os conceitos apresentados, o levantamento e a classificação da geodiversidade na bacia do Silva, foram realizados através dos dados de declividade, geologia, litologia, classes de solo e biótopos e hidrologia.

Através do software ArcGis 9.3 elaborou-se a análise espacial, que posteriormente foi trabalhada no SPSS, com a finalidade de agrupar os resultados homogêneos (cluster) deíndices de geodiversidade múltipla e ponderada.

Através do projeto “Geologia do Quadrilátero Ferrífero" , de autoria da Companhia de Desenvolvimento Econômico de Minas Gerais - CODEMIG, foram obtidos os shapefiles delitologia, geologia, estrutural, afloramento e minas.

O Instituto Mineiro de Gestão das Águas - IGAM, disponibilizou para fins de pesquisa acadêmica, a base hidrográfica ottocodificada e o estudo de regionalização de vazão.

Os dados referentes à classificação de solos foram disponibilizados pela Fundação Estadual do Meio Ambiente - FEAM. Neste caso deve ser ressaltado que o levantamento desolos foi realizado através da Universidade Federal de Viçosa, no ano 2010.

Os shapefiles de Curvas de nível de 20 em 20m, biótopos e unidades de conservação estão disponíveis na internet no domínio do geosisemanet.

A construção do banco de dados geográficos foi realizada através da delimitação da bacia hidrográfica na articulação da Carta Internacional ao Milionésimo, IBGE, quadrantesSF23-X-A-III-1 e SF-23-X-A-III-3 que correspondem respectivamente a Itabirito e a Casa dePedra.

Tabela 1: Banco de dados 


\begin{tabular}{|l|l|}
\hline \multicolumn{1}{|c|}{ SHAPEFILE } & \multicolumn{1}{c|}{ FONTE } \\
\hline Area da Bacia & IBGE \\
\hline Hidrografia & IGAM \\
\hline Geologia, litologia, estrutura, afloramentos & CODEMIG \\
\hline Biótopos, curvas de nivel & GEOSISEMA NET \\
\hline Classes de solo & FEAM \\
\hline
\end{tabular}

Para a pesquisa, foi construído o modelo digital de elevação - MDE, o mapa de toposde morro e o mapa de declividade da bacia hidrográfica. Para a construção do mapa de topos de morros foi observado o shapefile de curvas de nível e realizou-se a diferença entre a maior e a menor altitude do relevo. O resultado foi dividido em 03 classes, sendo considerada como topo de morro a classe mais elevada, ou seja, entre 1360 a 1560 metros.

Outro shapefile elaborado a partir das do modelo digital de elevação foi a declividadeacentuada, ou seja, maior que 45 graus.

No primeiro ensaio foi considerada a área total da bacia hidrográfica $95 \mathrm{~km}^{2}$, semdivisões. No segundo ensaio ocorreu a divisão da área em três grandes grupos: alto, médio e baixo Silva. No terceiro ensaio foi utilizada a divisão da bacia em 498 polígonos, de acordo com as áreas definidas na base hidrográfica ottocodificada.

O critério utilizado para a divisão da bacia hidrográfica em alto, médio e baixoribeirão do Silva, foi estabelecido em conformidade com as contribuições dos principais afluentes, a variação de altitude e a nomenclatura dos cursos d' água. Deste modo, considerouse: Alto Silva: área da nascente do ribeirão do Silva até a sua confluência com o ribeirão Aredes, abrangendo uma área de $38,75 \mathrm{Km}^{2}$; médio Silva: inicia na confluência do ribeirão do Silva com o ribeirão Aredes e finda no encontro com o ribeirão Mata-porcos, com área de 41,58 Km ${ }^{2}$; baixo Silva: inicia na confluência do ribeirão do Silva com o ribeirão Mataporcos, passando à denominação de ribeirão mata-porcos, até desaguar no ribeirão do Eixo com área de 14,74 Km 2 .

Após a construção dos dados geográficos, foi iniciada em sequência, a manipulaçãodas informações para a quantificação e identificação dos índices de geodiversidade. Foram realizados ensaios no ArcGis 9.3 e no SPSS para a sobreposição de imagens e agrupamento dos dados gerados.

Primeiramente foi realizada a junção das informações que estavam distribuídas emdiferentes camadas (layer) em uma única camada, constando a distribuição de todas as feições (variáveis) na área de estudo, distinguindo em classes, porém sem especificar. 
Para isto foi utilizada a ferramenta Spatialjoin, tendo como embasamento a área total da bacia do silva e atribuída os shapefiles com os elementos: litologia, geologia, espeleologia,afloramentos, solo, biótopos, estruturas, áreas de topo de morro, declividade acentuada $>45^{\circ}$ e hidrologia.

Como produto final foi gerado um shapefile, no qual a tabela de atributos consta a ocorrência das feições na área, como contagem simples, conforme exemplificado na tabela 2.

Tabela 2: Geodiversidade Específica

\begin{tabular}{|r|r|r|r|r|r|r|r|r|r|r|}
\hline Bacia & cavernas & afloram & geologia & estrutura & litologia & solo & biótopos & $\begin{array}{l}\text { decliv. } \\
>45^{\circ}\end{array}$ & $\begin{array}{r}\text { cursos } \\
\text { topos } \\
\text { d'água }\end{array}$ \\
\hline 0 & 40 & 6 & 14 & 400 & 19 & 5 & 331 & 732 & 105 & 485 \\
\hline
\end{tabular}

Em sequência, foram realizados os cálculos da área, da Geodiversidade Múltipla e daGeodiversidade Múltipla Ponderada, através da ferramenta Field Calculator, no ArcGis.

A geodiversidade múltipla foi obtida através do somatório da geodiversidade específica.

O cálculo da geodiversidade múltipla ponderada foi realizado através da divisão dageodiversidade múltipla pelo valor da área em quilômetros quadrados.

Todos os procedimentos citados foram realizados para as demais divisões da área, ouseja, alto, médio e baixo silva, além da divisão em 498 polígonos estabelecidos pela base hidrográfica ottocodificada.

Após a aplicação da metodologia proposta por XAVIER-DA-SILVA (2004), os dados gerados na planilha de atributos foram exportados para a aplicação do método de análise por agrupamento através do algoritmo "K-means", no software SPSS, com o objetivo de a agrupar categorias homogêneas, observando apenas a similaridade ou dissimilaridade entre estes.

O agrupamento realizado no SPSS foi novamente especializado no ArcGis 9.3 eatravés da ferramenta "SpatialStatistics", introduzido o "Mean Center", que identifica o centro geográfico para um conjunto de feições (centro de concentração).

A escolha do algoritmo K-means teve como referência o caráter não hierárquico para aobtenção dos grupos. Os métodos não hierárquicos são caracterizados pela facilidade computacional. A equação 1, representa este algoritmo. 


$$
J=\sum_{i=1}^{k} \sum_{i=1}^{n}\left\|x_{i}^{(i)}-c_{i}\right\|^{2}
$$

No qual, || $\mathrm{Xi}(\mathrm{j})-\mathrm{Cj}||^{2}$ corresponde ao cálculo da distância média entre um ponto dado $\mathrm{Xi}(\mathrm{j})$ e o centro do cluster $\mathrm{Cj}$.

Entretanto, antes de iniciar a execução é necessário a definição do número de grupos (clusters) que se pretende dividir a população de dados. Outra consideração sobre o método éque para um resultado efetivo, são necessárias várias iterações.

O SPSS possui esse algoritmo implementado, o que facilitou a aplicação desta etapa. Foi informado o número de grupos a ser formado, estipulado o número de iterações e a variável a ser agrupada. No primeiro ensaio foi agrupada a geodiversidade múltipla e no segundo, a geodiversidade múltipla ponderada.

\section{Resultados}

Os resultados obtidos através da sobreposição de informações espaciais e leitura das tabelas de atributos geradas durante o processo de construção podem ser interpretados como oíndice de geodiversidade específica, ou seja, a conta simples de quantas vezes cada feição (variável) está presente na área, conforme pode ser observado nas tabelas 3 e 4. 
Tabela 3: Geodiversidade Múltipla e Geodiversidade Múltipla Ponderada na bacia do Silva

\begin{tabular}{|l|r|}
\hline \multicolumn{1}{|c|}{ BACIA DO SILVA } & \multicolumn{1}{|c|}{ AREA TOTAL } \\
\hline espeleologia & 40 \\
\hline afloramentos & 6 \\
\hline geologia & 14 \\
\hline estrutura & 400 \\
\hline litologia & 19 \\
\hline solo & 5 \\
\hline biótopos & 331 \\
\hline declividade $>45^{\circ}$ & 732 \\
\hline topos & 105 \\
\hline cursos d'água & 485 \\
\hline Geodiversidade Múltipla & 2137 \\
\hline área $\left(\mathrm{Km}^{2}\right)$ & 95 \\
\hline Geodiversidade Múltipla Ponderada & 22,47581 \\
\hline
\end{tabular}

A interpretação da tabela está relacionada à quantidade de vezes que cada feiçãoocorre na área, sem distinguir as classes da variável. Assim, em toda a bacia do Silva ocorrem 40 feições espeleológicas, 06 afloramentos rochosos, 14 formações geológicas, 400 formações estruturais, 19 tipos de litologia, 05 classes de solo, 331 biótopos, 732 ocorrências de declividades acentuadas superiores a 45 graus, 105 topos de morro e 485 cursos d'água.

O somatório de todas as feições resulta em 2.137 variáveis, representando o índice de geodiversidade múltipla (XAVIER-DA-SILVA, 2004).

A ponderação da ocorrência da geodiversidade múltipla é realizada em relação àamplitude total $\mathrm{da}$ área, em quilômetros quadrados, e nomeada índice de geodiversidademúltipla ponderada. Deste modo, na bacia do silva esse índice corresponde àaproximadamente 23 variáveis.

Para a melhor visualização do modo como ocorre a distribuição das feições na área,foi utilizada a divisão da bacia em alto, médio e baixo Silva. Os resultados estão apresentados na tabela 4 e figura 3 . 
Tabela 4: Geodiversidade Múltipla e Geodiversidade Múltipla Ponderada na Alta, Média e Baixa bacia do ribeirão do Silva

\begin{tabular}{|l|l|l|l|}
\hline \multicolumn{1}{|c|}{ BACIA DO RIBEIRÃO DO SILVA } & \multicolumn{1}{c|}{ BAIXO } & \multicolumn{1}{c|}{ MÉDIO } & ALTO \\
\hline espeleologia & 1 & 23 & 16 \\
\hline afloramento & 4 & 1 & 1 \\
\hline geologia & 5 & 11 & 10 \\
\hline estrutura & 85 & 193 & 147 \\
\hline litologia & 12 & 13 & 17 \\
\hline solo & 3 & 3 & 4 \\
\hline biótopo & 8 & 170 & 177 \\
\hline declividade $>45^{\circ}$ & 135 & 364 & 235 \\
\hline altitude & 0 & 44 & 72 \\
\hline cursos d'água & 100 & 206 & 186 \\
\hline Geodiversidade Múltipla & 353 & 1028 & 865 \\
\hline Área Km ${ }^{2}$ & 14,74 & 41,58 & 38,75 \\
\hline Geodiversidade Múltipla Ponderada & 23,95 & 24,72 & 22,32 \\
\hline
\end{tabular}

Interpretando a tabela 4, foi observado que a variação do índice de geodiversidademúltipla é mais elevado no médio ribeirão do Silva, podendo estar relacionado com drenagem, uma vez que a área apresenta 206 cursos d' água. A declividade, as estruturas e os biótopos podem ser caracterizados como fatores relevantes. Ainda no contexto, é a área mais ampla, o que justificaria a elevação deste índice. Entretanto, após a ponderação em função daárea, resultando na geodiversidade múltipla ponderada, o índice permaneceu elevado em relação ao alto e baixo ribeirão do Silva. 


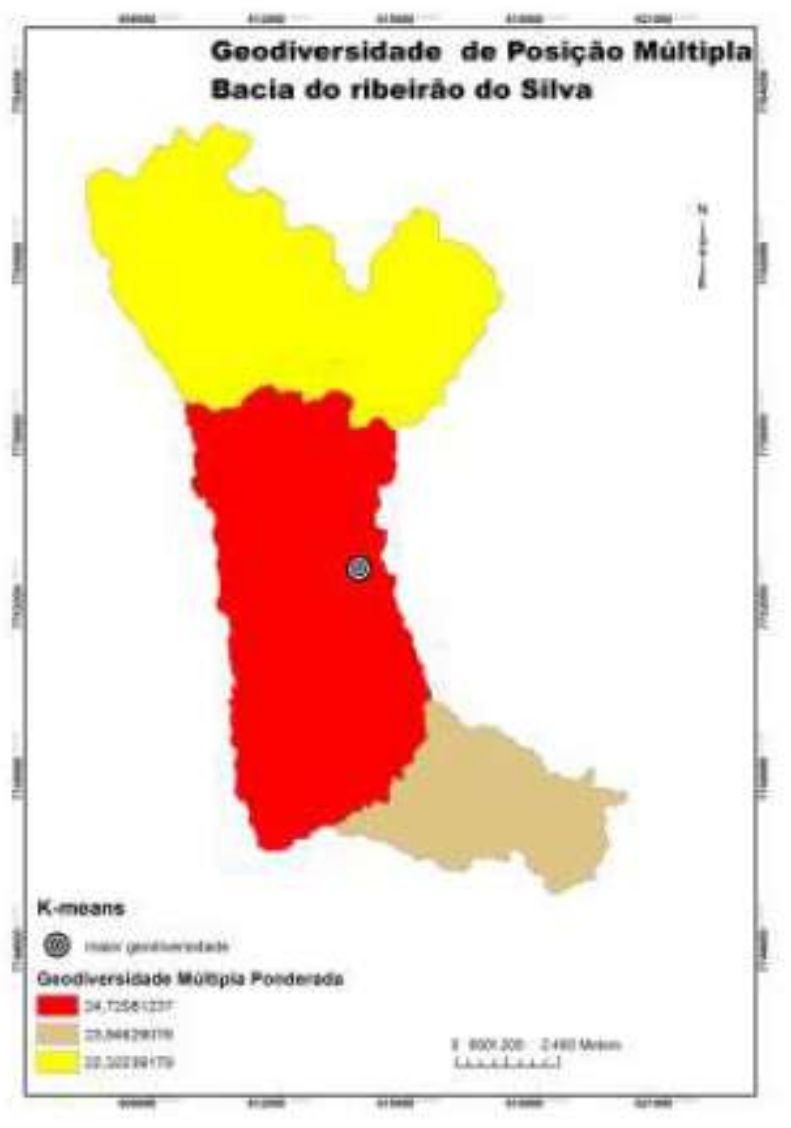

Figura 3: Índice de geodiversidade de posição no alto, médio e baixo Silva

Com respaldo nos resultado apresentados, foi elaborado o mapa de geodiversidade de posição de múltipla e a identificação do ponto onde ocorre o índice de maior frequência da geodiversidade múltipla, podendo ser observado na figura 4 . 


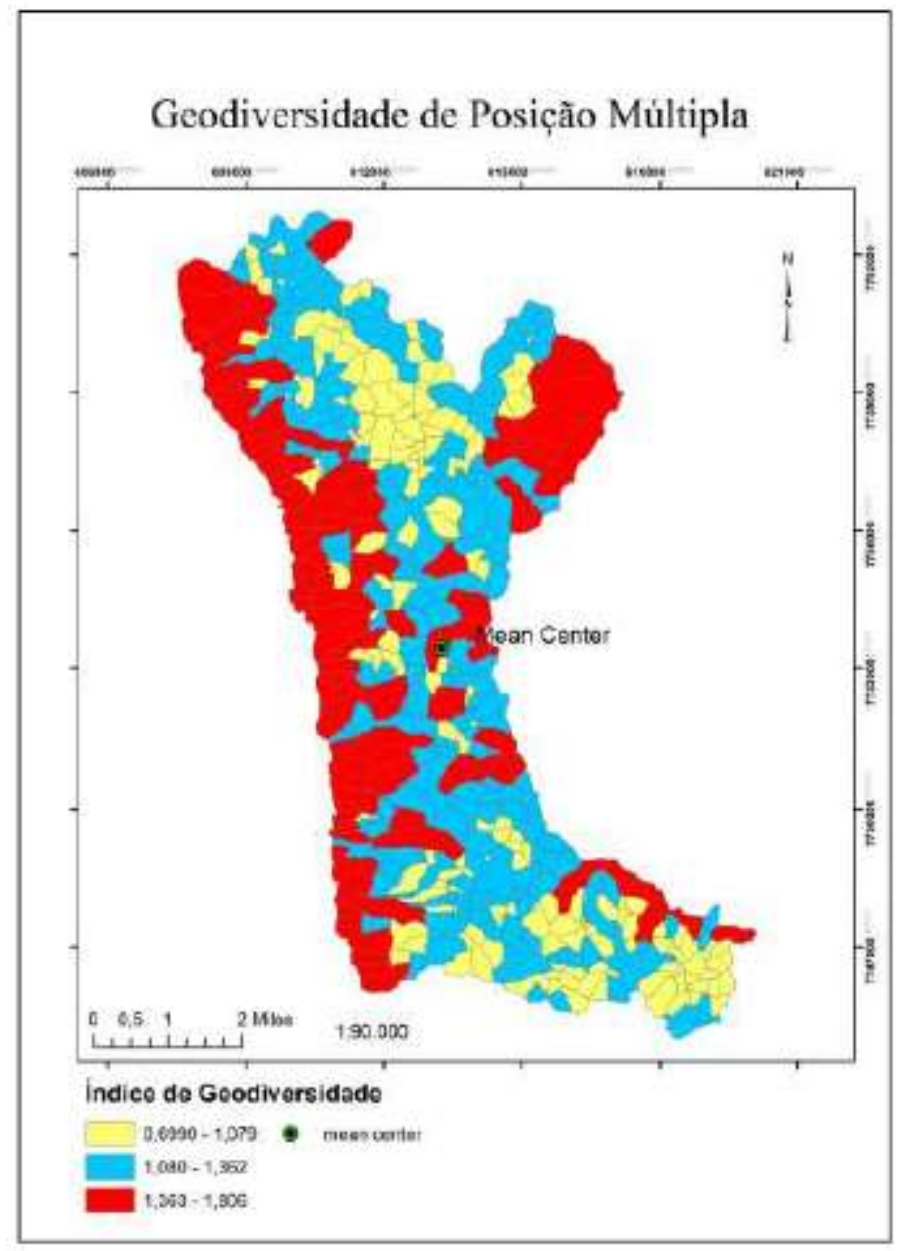

Figura 4: Geodiversidade de Posição Múltipla da bacia do Silva

Análise por agrupamento "K-means"

Com objetivo de alcançar o resultado que mais se aproxima da realidade local, foi realizada a análise por agrupamento através do algoritmo “K-means”. Os dados geográficosarmazenados nas tabelas de atributos foram trabalhados no SPSS. Nesta etapa foi utilizada adivisão da base hidrográfica ottocodificada, considerando os 498 polígonos, que compreendem toda a área da bacia hidrográfica.

Como os polígonos não são divididos em áreas de mesma grandeza, foram utilizadosas variáveis: índices de geodiversidade múltipla e geodiversidade múltipla ponderada. O número de iterações em cada caso foram 100, além de ter sido indicado a formação de 3 grupos. Entretanto, a indicação de 03 grupos não foi satisfatória para o índice degeodiversidade múltipla ponderada e repetiu-se a operação com a formação de 10 grupos, conforme pode ser observado nos resultados. 
a) Resultado da análise por agrupamento "K-means" do índice de geodiversidade múltipla

Final Cluster Centers

\begin{tabular}{|c|c|c|c|}
\hline \multirow{2}{*}{} & \multicolumn{3}{|c|}{ Cluster } \\
\cline { 2 - 4 } & 1 & 2 & 3 \\
\hline geodiv & 40,25000000 & 10,92465753 & 20,59602649 \\
\hline
\end{tabular}

Distances between Final Cluster Centers
\begin{tabular}{|l|r|r|r|}
\hline Cluster & \multicolumn{1}{|c|}{1} & \multicolumn{1}{c|}{2} & \multicolumn{1}{c|}{3} \\
\hline 1 & & 29,325 & 19,654 \\
2 & 29,325 & & 9,671 \\
3 & 19,654 & 9,671 & \\
\hline
\end{tabular}

Final da iteração K-means da geodiversidade Múltipla:

Convergência alcançada devido à falta ou pequena mudança nos centros de cluster

A variação máxima de coordenadas absoluta para qualquer centro: 000 .

Número máximo de iterações: 12.

Distância mínima entre os centros iniciais: 26.000 .

\begin{tabular}{|c|c|c|c|c|c|c|}
\hline & \multicolumn{2}{|c|}{ Cluster } & \multicolumn{2}{|c|}{ Error } & \multirow[b]{2}{*}{ F } & \multirow[b]{2}{*}{ Sig. } \\
\hline & Mean Square & df & Mean Square & df & & \\
\hline geodiv & 21495,849 & 2 & 14,325 & 496 & 1500,583 &, 000 \\
\hline
\end{tabular}

Number of Cases in each Cluster

\begin{tabular}{|ll|r|}
\hline Cluster & 1 & 56,000 \\
& 2 & 292,000 \\
& 3 & 151,000 \\
& 3 & 499,000 \\
Valid & &, 000 \\
Missing & & \\
\hline
\end{tabular}


b) Resultado da análise por agrupamento "K-means" do índice de geodiversidade múltipla ponderada

Final Cluster Centers

\begin{tabular}{|c|c|c|c|c|c|c|}
\hline \multirow{2}{*}{} & \multicolumn{7}{|c|}{ Cluster } \\
\cline { 2 - 8 } & 1 & 2 & 3 & 4 & 5 & 6 \\
\hline geo pond & 549900,0000 & 7666,0000 & 1551,3000 & 45150,0000 & 10793,3333 & 263900,0000 \\
\hline & \multicolumn{7}{|c|}{ Cluster } & & \\
& 7 & 8 & 9 & 10 & \\
\hline geo pond & 638,6667 & 3384,3333 & 6016,5000 & 118,5425 \\
\hline
\end{tabular}

b. Final da iteração K-means da geodiversidade Múltipla ponderada:

Convergência alcançada devido à falta ou pequena mudança nos centros de cluster.

A variação máxima de coordenadas absolutas para qualquer centro é, 000 .

Número máximo de iterações: 33.

A distância mínima entre os centros iniciais é 938000.

Distances between Final Cluster Centers

\begin{tabular}{|l|r|r|r|r|r|r|}
\hline Cluster & \multicolumn{1}{|c|}{1} & \multicolumn{1}{c|}{2} & \multicolumn{1}{c|}{3} & \multicolumn{1}{c|}{4} & \multicolumn{1}{c|}{5} & \multicolumn{1}{c|}{6} \\
\hline 1 & & 41765,667 & 218750,000 & 39133,500 & 43165,500 & 44044,044 \\
2 & 41765,667 & & 260515,667 & 2632,167 & 1399,833 & 2278,378 \\
3 & 218750,000 & 260515,667 & & 257883,500 & 261915,500 & 262794,044 \\
4 & 39133,500 & 2632,167 & 257883,500 & & 4032,000 & 4910,544 \\
5 & 43165,500 & 1399,833 & 261915,500 & 4032,000 & & 878,544 \\
6 & 44044,044 & 2278,378 & 262794,044 & 4910,544 & 878,544 & \\
7 & 44811,519 & 3045,852 & 263561,519 & 5678,019 & 1646,019 & 767,474 \\
8 & 37484,000 & 4281,667 & 256234,000 & 1649,500 & 5681,500 & 6560,044 \\
9 & 34356,667 & 7409,000 & 253106,667 & 4776,833 & 8808,833 & 9687,378 \\
10 & 504750,000 & 546515,667 & 286000,000 & 543883,500 & 547915,500 & 548794,044 \\
\hline
\end{tabular}

Distances between Final Cluster Centers

\begin{tabular}{|l|r|r|r|c|}
\hline Cluster & \multicolumn{1}{|c|}{7} & \multicolumn{1}{c|}{8} & \multicolumn{1}{c|}{9} & \multicolumn{1}{c|}{10} \\
\hline 1 & 44811,519 & 37484,000 & 34356,667 & 504750,000 \\
2 & 3045,852 & 4281,667 & 7409,000 & 546515,667 \\
3 & 263561,519 & 256234,000 & 253106,667 & 286000,000 \\
4 & 5678,019 & 1649,500 & 4776,833 & 543883,500 \\
5 & 1646,019 & 5681,500 & 8808,833 & 547915,500 \\
6 & 767,474 & 6560,044 & 9687,378 & 548794,044 \\
7 & & 7327,519 & 10454,852 & 549561,519 \\
8 & 7327,519 & & 3127,333 & 542234,000 \\
9 & 10454,852 & 3127,333 & & 539106,667 \\
10 & 549561,519 & 542234,000 & 539106,667 & \\
\hline
\end{tabular}




\begin{tabular}{|c|c|c|c|c|c|c|}
\hline \multicolumn{7}{|c|}{ ANOVA } \\
\hline & \multicolumn{2}{|c|}{ Cluster } & \multicolumn{2}{|c|}{ Error } & \multirow[b]{2}{*}{$\mathrm{F}$} & \multirow[b]{2}{*}{ Sig. } \\
\hline & Mean Square & df & Mean Square & df & & \\
\hline geo pond & $3,836 \mathrm{E} 10$ & 9 & 73914,632 & 20 & 518997,574 &, 000 \\
\hline
\end{tabular}

\begin{tabular}{|c|c|c|}
\hline \multicolumn{3}{|c|}{$\begin{array}{c}\text { Number of Cases in each } \\
\text { Cluster }\end{array}$} \\
\hline \multirow[t]{10}{*}{ Cluster } & 1 & 1,000 \\
\hline & 2 & 1,000 \\
\hline & 3 & 10,000 \\
\hline & 4 & 1,000 \\
\hline & 5 & 3,000 \\
\hline & 6 & 1,000 \\
\hline & 7 & 30,000 \\
\hline & 8 & 3,000 \\
\hline & 9 & 2,000 \\
\hline & 10 & 447,000 \\
\hline Valid & & 499,000 \\
\hline Missing & &, 000 \\
\hline
\end{tabular}

As análises por agrupamento através do algoritmo “K-means” resultaram na formação de 3 grupos homogêneos para o índice de geodiversidade múltipla, com 100 iterações, findando, em todas as vezes que foi rodado, na décima segunda tentativa. A melhor indicação foi a formação de 2 grupos, conforme pode ser observado no teste de hipótese realizado.

Para o índice de geodiversidade múltipla ponderada, o melhor resultado foi através daindicação de 9 grupos, conforme pode ser observado nos resultados dos testes de hipóteses gerados através do SPSS .

Deve ser ressaltado que aplicação dos testes de hipótese foram realizados apenas parafins de descrição, os grupos determinados apresentam apenas a maximização das diferenças entre os casos de dissimilaridade. Os níveis de significância observados não são corrigidos e, portanto, não podem ser interpretados como provas da hipótese de que os meios de fragmentação são iguais.

Em consenso com a explicação sobre os testes de hipótese, foi respeitado a formação de 03 grupos para o índice de geodiversidade múltipla e 10 grupos para o índice de geodiversidade múltipla ponderada. 
Os resultados dos agrupamentos foram especializados, gerando, como produto final os mapas de grupos de geodiversidade múltipla e ponderada. Para a identificação do ponto que apresenta o maior conjunto de geodiversidade, foi acrescentado o "mean center".

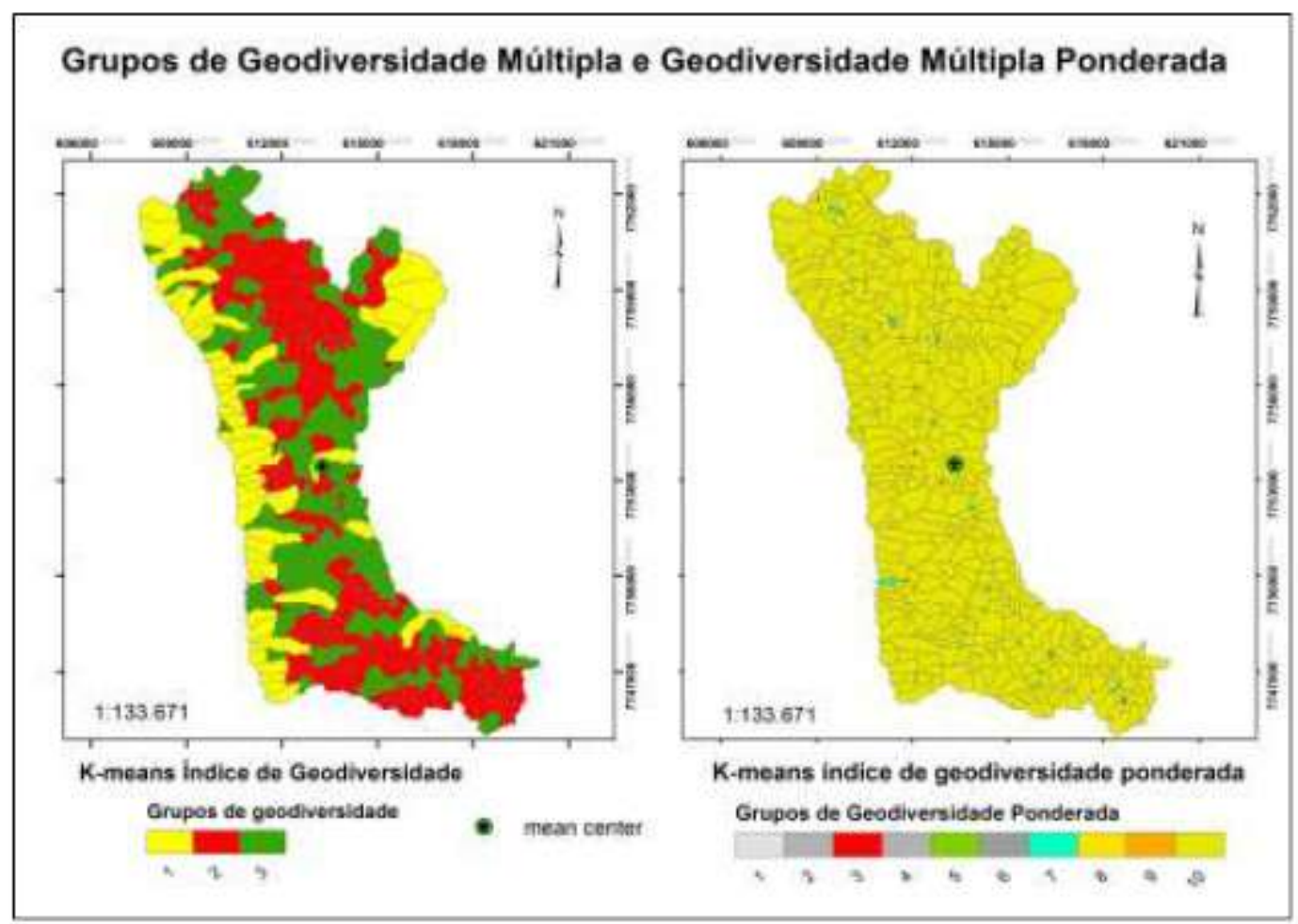

Figura 6: Análise por agrupamento "K-means"

Comparando os resultados das figuras 05 e 06 conclui-se que o método de análise poragrupamento, através do algoritmo "K-means" , pode ser trabalhado com eficiência para nas análises estatísticas espaciais dos índices de geodiversidade para pequenas áreas, como foi demonstrado através do estudo realizado na bacia do Silva.

O resultado alcançado poderá subsidiar o desenvolvimento de novas pesquisas na áreaou em pequenos recortes espaciais, que objetivem a conservação da geodiversidade local, a tomada de decisão de gestores, o desenvolvimento de planos de gestão da bacia hidrográfica, além da criação de Unidades de Conservação.

\section{Considerações}

A metodologia aplicada neste trabalho foi proposta para grandes áreas, para aaplicação em levantamentos estaduais, utilizando como polígonos os limites político-administrativos. Entretanto, esta metodologia pode ser bem aplicada em áreas menores. 
A escala da base de dados geográficos deve ser observada, de modo que a análiseespacial seja efetiva, demonstrando a realidade local.

Outras técnicas para a quantificação da geodiversidade para pequenas áreas devem serdesenvolvidas para dar suporte aos gestores e auxiliar na conservação do meio físico e do patrimônio geológico.

\section{Bibliografia}

BRILHA, J. 2005. Patrimônio Geológico e Geoconservação: A conservação da Natureza na sua vertente geológica. Braga, Palimage Editores, 190 p.

CODEMIG. Programa de Mapeamento Geológico Quadrilátero. Ferrífero http://www.codemig.com.br/site/content/parcerias/levantamento_aerogeofisico.asp?id=30\&i $\mathrm{dSubPrj}=50 \&$ filhoId $=62$ acesso em 01/11/2012

FILHO, D. B. F.; JÚNIOR, J. A. S.; ROCHA, E. C. Classificando Regimes Políticos Utilizando Análise de Conglomerados. Opinião Pública, Campinas, vol. 18, $\mathrm{n}^{\mathrm{O}}$ 1, Junho, 2012, p. 109 128

HAIR, Jr; BLACK, W. C; BABIN, B. J.; ANDERSON, R. E.; TATHAM, R. L. Análise Multivariada de Dados. Porto Alegre: Bookman, 2005.

JESUS, N. B.; CARDOSO, M. G. M. S. Análise de Agrupamento incremental - Segmentação de Pontos de Retalho. Revista de Ciências da Computação, Volume II, Ano II, 2007, n ${ }^{\circ} 2$

MANOSSO, F.C.; ONDICOL, R. P. Geodiversidade: Considerações Sobre Quantificação eAvaliação da Distribuição Espacial. Anuário do Instituto de Geociências. UFRJ. Vol. 351/2012. P.90-100

NUNES, A.; BARROS, E. Portuguese Regional UnemploymentPatterns: A k-Means Cluster Analysis Approach. OvidiusUniversityAnals, EconomicSciences Series. Volume X, 2010

SOLÁ, M. E. C. 2008. In: Patrimônio natural: cultural e zoneamento ecológico econômico da Serra da Moeda: uma contribuição para sua conservação. Organização e edição: Maria Elisa Castellanos Solá; Patrimônio Arqueológico e Histórico: Carlos Magno Guimarães (Coord.); Patrimônio Histórico, Arquitetônico, Urbanístico e Paisagístico: JoséEustáquio Machado Paiva (Coord.). Belo Horizonte: Brandt Meio Ambiente, 2008. 2 v. + 1CD-ROM. 